\title{
SETS THAT REMAIN MAXIMAL MONOTONE UNDER ALL MONOTONE PERTURBATIONS
}

\author{
BRUCE CALVERT
}

\begin{abstract}
We characterize maximal monotone sets whose domain has nonempty interior and which remain maximal monotone under all perturbations.
\end{abstract}

Let $H$ be a real Hilbert space and let $A$ be a maximal monotone set with open domain $D(A)$. From [1] it follows that if $B$ is maximal monotone such that $D(B) \cap D(A) \neq \varnothing$ then $A+B$ is maximal monotone; i.e., $A$ remains maximal monotone under all maximal monotone perturbations $B$ satisfying $D(A) \cap D(B) \neq \varnothing$.

At the Fourth C.I.M.E. Session, Varenna, 1970, A. Pazy raised the question of the characterization of such maximal monotone sets $A$. In the present note we answer Pazy's question for the special case of maximal monotone sets $A$ satisfying $\operatorname{int}(D(A)) \neq \varnothing$.

Let $I$ be the set of maximal monotone $A$ satisfying $\varnothing \neq$ interior $D(A) \neq$ $D(A)$. Let $P$ be the set of sets $A$ that remain maximal monotone under all perturbations. Let $M$ be the set of maximal monotone sets $A$ having the property that for $x$ in $D(A)$ and $N$ a supporting closed hyperplane to $D(A)$ at $x, P_{N}(A x)=N$, where $P_{N}$ is the orthogonal projection on $N$.

THEOREM. $\varnothing \neq P \cap I=M \cap I \subsetneq I$. Also, $P \subset M$.

Proof. We show first that $M \cap I \subset P$. Suppose $A \in M \cap I$. Suppose $B$ is maximal monotone and $D(B) \cap D(A) \neq \varnothing$. If $D(B) \cap$ int $D(A) \neq \varnothing$, then $A+B$ is maximal by [1]. If not, there exists $x$ in $D(B) \cap D(A)-$ int $D(A)$. Since $\operatorname{cl}(D(A)), \operatorname{cl}(D(B))$ are convex and int $D(A) \not \equiv \varnothing$ there is a closed hyperplane $N$ separating $D(A)$ and $D(B)$. Take $y$ in $H$ such that $N=\{z \mid(z, y)=(x, y)\}$ and $(a, y) \leqq(x, y)$ for $a$ in $D(A)$ and $(b, y) \geqq(x, y)$ for $b$ in $D(B)$. Take $\left[x, x^{*}\right]$ in $B$. For $\left[b, b^{*}\right]$ in $B$, and $k \geqq 0,\left(x^{*}-b^{*}-k y\right.$, $x-b) \geqq 0$. By the maximality of $B, B x=\{B x-k y: k \geqq 0\}$. Similarly, $A x=\{A x-k y: k \leqq 0\}$. Since $P_{N}(A x)=N, A x+B x=H$. Hence, $A+B=$ $\{[x, z]: z \in H\}$ giving $A+B$ maximal monotone, and proving $M \cap I \subset P$.

We now show $P \subset M$. We suppose $A$ is maximal monotone but not in $M$.

Received by the editors June 8, 1973.

AMS (MOS) subject classifications (1970). Primary 47Hxx.

Key words and phrases. Monotone operators, perturbation.

(C) American Mathematical Society 1974 
By translation we may suppose 0 is in $D(A), x_{0}$ is in $H, D(A) \subset\left\{w \mid\left(w, x_{0}\right) \leqq\right.$ $0\}$, and $P_{N} A(0) \neq N$ where $N=\left\{w \mid\left(w, x_{0}\right)=0\right\}$. Define $B$ by $D(B)=\{z$ : $\left.\left(z, x_{0}\right)>0\right\} \cup\{0\}, \quad B(0)=\left\{-k x_{0}: k \geqq 0\right\}, \quad$ and if $\left(z, x_{0}\right)>0, \quad B(z)=z+$ $\left(z, x_{0}\right) x_{0}-(z, z)\left(z, x_{0}\right)^{-1} x_{0} . B$ is maximal monotone by Lemma 1 below, but $D(A+B)=\{0\}$ and $(A+B)(0) \subseteq\left\{A(0)+k x_{0}: k\right.$ real $\} \neq H$ since $P_{N} A(0) \neq$ $N$.

\section{Lemma $1 . \quad B$ defined above is maximal monotone.}

Proof. For $z$ satisfying $\left(z, x_{0}\right)>0, B z$ may be described as follows. Consider a two-dimensional vector space containing $x_{0}$ and $z$, and suppose $x_{0}$ has coordinates $(1,0)$ and $z$ has coordinates $(x, y)$. Then $B z=$ $\left(\left(x^{2}-y^{2}\right) / x, y\right)$. We show $B$ is monotone. Suppose $\left(z_{1}, x_{0}\right)>0$ and $\left(z_{2}, x_{0}\right)>$ 0 ; then we take a three-dimensional vector space containing $x_{0}, z_{1}$ and $z_{2}$. In this space, $R^{3}$, for $z=(d, e, f)$ in $R^{3}(d>0)$, we have $B z=\left(d-\left(e^{2}+f^{2}\right) / d\right.$, $e, f)$. If $z_{1}=\left(d_{1}, e_{1}, f_{1}\right)$ and $z_{2}=\left(d_{2}, e_{2}, f_{2}\right)$,

$$
\begin{aligned}
\left(B z_{2}-B z_{1}, z_{2}-z_{1}\right)= & \left(d_{2}-d_{1}\right)^{2}+\left(e_{2}-e_{1}\right)^{2}+\left(f_{2}-f_{1}\right)^{2} \\
& -\left(d_{2}-d_{1}\right)\left(\left(e_{2}^{2}+f_{2}^{2}\right) / d_{2}-\left(e_{1}^{2}+f_{1}^{2}\right) / d_{1}\right) \\
= & \left(d_{2}-d_{1}\right)^{2}-2 e_{1} e_{2}-2 f_{1} f_{2} \\
& +\left(e_{1}^{2}+f_{1}^{2}\right) d_{2} / d_{1}+\left(e_{2}^{2}+f_{2}^{2}\right) d_{1} / d_{2} .
\end{aligned}
$$

Using $2 a b \leqq a^{2}+b^{2}$, where $a=e_{1}\left(d_{2} / d_{1}\right)^{1 / 2}$ and $b=e_{2}\left(d_{1} / d_{2}\right)^{1 / 2}$, etc., gives the right-hand side $\geqq\left(d_{2}-d_{1}\right)^{2}$. To complete the proof that $B$ is monotone we take $z_{1}$ with $\left(x_{0}, z_{1}\right)>0$ and $z_{2}=0$.

$$
\begin{aligned}
\left(B z_{2}-\right. & \left.B z_{1}, z_{2}-z_{1}\right) \\
& =\left(-k x_{0}-z_{1}-\left(z_{1}, x_{0}\right) x_{0}+\left(z_{1}, z_{1}\right)\left(z_{1}, x_{0}\right)^{-1} x_{1},-z_{1}\right), \quad k>0, \\
& =k\left(x_{0}, z_{1}\right)+\left(z_{1}, x_{0}\right)^{2} .
\end{aligned}
$$

We show $B$ is maximal. To show $R(1+B)=H$ it is enough to show that the operator $B$ in $R^{2}$ described at the beginning of the lemma satisfies $R(1+B)=R^{2}$. Given $(u, v)$ in $R^{2}$, if $v=0, u \leqq 0$, then $(I+B)(0,0)$ contains $(u, v)$, otherwise we take $(x, y)$ satisfying $2 x-y^{2} / x=u$, and $2 y=v$. Q.E.D.

We show that $P \cap I \neq I$ by noting that $B$ constructed in Lemma 1 is in $I$ but not in $P$.

We show $P \cap I \neq \varnothing$ by the following result, completing the proof of the Theorem.

Lemma 2. Take $x_{0}$ in $H$, of norm 1 , and for $z$ satisfying $\left(x_{0}, z\right)>0$, let $A z=2\left(z, x_{0}\right)^{-1} z-(z, z)\left(z, x_{0}\right)^{-2} x_{0}-x_{0}$. Let $A(0)=\left\{z: 4\left(z, x_{0}\right)+(z, z)-\right.$ $\left.\left(z, x_{0}\right)^{2} \leqq 0\right\}$. Otherwise let $A z=\varnothing$. The $A$ is in $M \cap I$. 
Proof. In a two-dimensional space $R^{2}$ containing $x_{0}=(1,0)$ we have, if $z=(x, y), x>0, \quad A z=\left(-y^{2} / x^{2}, 2 y / x\right), \quad$ and $A(0) \cap R^{2}=\{(u, v): 4 u+$ $\left.v^{2} \leqq 0\right\}$. With $N=\left\{z:\left(z, x_{0}\right)=0\right\}$, the only hyperplane supporting $D(A)$, since $N \cap R^{2}$ is the set $\{(0, v): v$ in $R\}$, we have $P_{N} A(0)=N$.

We show $A$ is monotone. Take $z_{1}$ and $z_{2}$ such that $\left(z_{1}, x_{0}\right)>0$ and $\left(z_{2}, x_{0}\right)>0$. Taking a three-dimensional vector space containing $x_{0}, z_{1}$, and $z_{2}$, we have, for $x_{0}=(1,0,0)$ and $z=(d, e, f), d>0$, that $A z=$ $\left(-\left(e^{2}+f^{2}\right) / d^{2}, 2 e / d, 2 f / d\right)$. If $z_{1}=\left(d_{1}, e_{1}, f_{1}\right)$ and $z_{2}=\left(d_{2}, e_{2}, f_{2}\right)$ then

$$
\begin{aligned}
\left(A z_{2}-A z_{1}, z_{2}-z_{1}\right)= & \left(-\left(e_{2}^{2}+f_{2}^{2}\right) / d_{2}^{2}+\left(e_{1}^{2}+f_{1}^{2}\right) / d_{1}^{2}\right)\left(d_{2}-d_{1}\right) \\
& +\left(e_{2} / d_{2}-e_{1} / d_{1}\right)\left(d_{2}-d_{1}\right) \\
& +\left(2 e_{2} / d_{2}-2 e_{1} / d_{1}\right)\left(e_{2}-e_{1}\right) \\
& +\left(2 f_{2} / d_{2}-2 f_{1} / d_{1}\right)\left(f_{2}-f_{1}\right) .
\end{aligned}
$$

Using $2 a b \leqq a^{2}+b^{2}$, where $a=e_{1} d_{2}^{1 / 2} / d_{1}$ and $b=e_{2} / d_{2}^{1 / 2}$, etc., gives the righthand side $\geqq 0$. To complete the proof that $A$ is monotone we consider $z_{1}$ with $\left(x_{0}, z_{1}\right)>0$, and $z_{2}=0$. In $R^{2}$, if $z_{1}=(x, y)$, then if $(u, v) \in A(0)$,

$$
\begin{aligned}
\left(A z_{1}-A z_{2}, z_{1}-z_{2}\right) & =\left(-y^{2} / x^{2}-u\right) x+(2 y / x-v) y \\
& \geqq-x\left(u+v^{2} / 4\right) \geqq 0,
\end{aligned}
$$

using $2 a b \leqq a^{2}+b^{2}$ with $a=(x / 2)^{1 / 2} v$ and $b=y(x / 2)^{-1 / 2}$.

To show $R(1+A)=H$, as in the proof of Lemma 1 it suffices to show that for $A$ in $R^{2}, R(I+A)=R^{2}$. Let $(u, v) \in R^{2}$. If $4 u+v^{2}>0$ then there is a unique $x>0$ such that $u=x-v^{2}(2+x)^{-2}$. Choosing $y=v x(2+x)^{-1}$ we have $(u, v)=(I+A)(x, y)$. If $4 u+v^{2} \leqq 0$ then $(u, v) \in(I+A)(0,0)$ and the proof is complete.

\section{REFERENCE}

1. R. Rockafellar, On the maximality of sums of nonlinear monotone operators, Trans. Amer. Math. Soc. 149 (1970), 75-88. MR 43 \#7984.

Department of Mathematics, University of Auckland, Auckland, New ZeaLAND 